\title{
NOTAS SOBRE LOS SUFIJOS AUMENTATIVOS EN EL ESPAÑOL DE LA ARGENTINA
}

\author{
Laura Kornfeld \\ Universidad Nacional de General Sarmiento \\ Consejo Nacional de Investigaciones Científicas y Técnicas
}

Resumen: Nos proponemos indagar en las propiedades de adjetivos y nombres formados a partir de los sufijos apreciativos -ón, -azo, -ote, los más usados con valor aumentativo en el español de Argentina.

Cuando modifican a nombres, el significado de los sufijos es siempre aumentativo, más allá de si se designan objetos (camperón, sillota), personas (minón, hombrote) o eventos (notición, estresazo). En cambio, cuando se adjuntan a adjetivos, los sufijos -ón y, en menor medida, -ote no aportan necesariamente significado aumentativo, sino afectivo (cfr. tristón, dulzón, seriote).

Intentamos sistematizar los valores $y$ las restricciones en la distribución de ón, -azo, -ote, así como sus relaciones con otros afijos apreciativos, además de explicar su naturaleza funcional o léxica desde un marco generativo.

Palabras clave: sufijos aumentativos, morfología apreciativa, español de la Argentina.

\begin{abstract}
This paper intends to explore the properties of adjectives and nouns created with -on, -azo, -ote, the most frequent suffixes with augmentative value in Argentina Spanish.

When modifying nouns, the meaning of these suffixes is always augmentative, independently of whether the nouns refer to objects (camperón, sillota), persons (minón, hombrote) or events (notición, estresazo). On the contrary, when adjoining adjectives, suffixes -ón and, to a lesser degree, -ote do not always add augmentative, but rather affective, meanings (cf. tristón, dulión, seriote). Our aims are to systematize the meanings and restrictions in the distribution of -ón, $-a z 0,-o t e$ and their relations with other appreciative affixes, and also to explain from a generative framework their functional or lexical nature.
\end{abstract}

Keywords: augmentative suffixes, appreciative morphology, Argentina Spanish. 


\section{Introducción}

En cada lengua encontramos un subconjunto significativo de recursos léxicos y gramaticales que los hablantes privilegian cuando pretenden expresar su universo subjetivo, sus pensamientos, sentimientos y sensaciones. Dentro de los recursos que se usan para codificar esa función del lenguaje (que podemos llamar emotiva, para adoptar la tradicional terminología de Jakobson en "Lingüística y poética") se encuentran dominios gramaticales como la cuantificación y la modalidad, además de los recursos deícticos que remiten en forma directa a la situación de enunciación. De hecho, podríamos ligar todos esos recursos con la noción de aparato formal de la enunciación, que deja entrever las huellas de que el hablante se ha apropiado del sistema de la lengua, en el sentido igualmente clásico de Benveniste (1974). En el caso del español, la morfología apreciativa guarda, indudablemente, una relación muy estrecha con los ámbitos de la cuantificación y la modalidad, como se percibe en ciertos fenómenos puntuales que los atraviesan, como el prefijo re- (según lo analizado en Kornfeld \& Kuguel, 2013) o el afijo diminutivo -it(o/a) (Kornfeld, 2015).

$\mathrm{Al}$ contrario del diminutivo, que tiene su representante casi exclusivo en el afijo o sufijo -it(o/a), ya que las realizaciones alternativas $-u c h(o / a),-i n(a)$ y $-i n(a)$ son mucho menos frecuentes, el significado descripto habitualmente como aumentativo posee al menos tres realizaciones productivas en el español de Argentina: los sufijos apreciativos -ón(a), - 
az $(0 / a)$, -ot(e/a $)^{1}$, que alternan para dar lugar a nombres $y$ adjetivos como los de $(1)^{2}$ :

(1) a. camperón, apuradón, asustadón, payasón, sorpresón, miedón, patadón

b. locazo, tontazo, tranquilazo, librazo, madraza, calorazo, miedazo, sustazo

c. grandote, animalote, bocota, orejota, camperota, piezota, sonrisota, manota

El presente trabajo se propone, justamente, indagar en las propiedades gramaticales y semánticas de los adjetivos y nombres formados a partir de esos tres sufijos. En tanto sufijos apreciativos, los tres son considerados procedimientos esencialmente homogéneos que nunca modifican la clase de palabra y que no se diferencian en forma tajante de la flexión ni desde el punto de vista semántico (ya que no dan lugar, en principio, a una nueva entrada de diccionario) ni desde el punto de vista estrictamente morfológico (siempre aparecen como el último proceso derivativo antes de la flexión). Esas

\footnotetext{
${ }^{1}$ De aquí en adelante, por razones de economía, usaremos solo la forma masculina de los sufijos.

${ }^{2}$ Los ejemplos presentados en este trabajo son utilizados (con muy diversos grados de lexicalización) en el español de la Argentina, aunque en muchos casos son compartidos con otras variedades del español. Solo se han incluido ejemplos que tengan registradas varias apariciones en el Google. Al mismo tiempo, se ha procurado evitar todos los casos de lexicalización que dan lugar a nuevas entradas en el diccionario (palabrota, comadrona, corralón, por ejemplo).
} 
propiedades, de hecho, han llevado a proponer reiteradamente que la morfología apreciativa tiene un estatuto intermedio entre la flexión y la derivación (cfr. Zacarías Ponce de León (2008) para una revisión minuciosa de esa hipótesis).

En ese panorama, tiene sentido analizar las propiedades gramaticales y semánticas de los sufijos de significado aumentativo en relación con el inventario completo de afijos apreciativos, en particular, el diminutivo -ito (al que algunas veces se oponen y otras se asemejan) y los diversos prefijos que son semánticamente análogos, como es el caso de re-, super-, hiper-, mega-, ultra-, también de valor aumentativo. Desde la perspectiva de la sintaxis formal, y, en particular, del modelo de la Morfología Distribuida de Marantz (2001) y Embick \& Halle (2011), uno de los tópicos relevantes que plantea la morfología apreciativa (y que recuperamos en este artículo) refiere a distinguir cuándo esa relación se codifica en rasgos formales alojados en proyecciones funcionales de la sintaxis (como pueden ser el Sintagma de Grado o algún sintagma ligado con la modalidad) y cuándo, en cambio, aparece expresada como un elemento ligado con las capas léxicas.

\section{Descripción de las formas}

En esta sección reseñamos las principales características gramaticales y semánticas de los sufijos aumentativos -ote, -azo y -ón, en su combinación con nombres y con adjetivos. 
Dado que los sufijos aumentativos son polisémicos, debemos primeramente circunscribir nuestro objeto de estudio en este trabajo. En lo que hace a la creación de nombres, se ha señalado a menudo que -ón y -azo producen alternativamente formas con un significado fundamentalmente apreciativo, como los de (1.a-b), o con uno eventivo. En este trabajo nos centramos solo en el primer caso, por lo que no tomamos en cuenta las nominalizaciones que resultan de la adjunción de -ón y -azo a verbos, como en (2.a), o a nombres no eventivos que, sin embargo, terminan dando lugar a formas intrínsecamente eventivas (que se interpretan como 'golpe (físico o político) dado con/por N', o con significados aún más lexicalizados), como ocurre con los ejemplos de (2.b):

(2) a. resbalón, raspón, apretón, empujón, apagón, frenazo, arañazo, cagazo, patinazo

b. piedrazo, perejilazo, viejazo, cuartelazo, papelón, plantón

En cambio, sí analizamos los casos en que la eventividad del nombre resultante es producto del carácter eventivo de la base, como en (3), ya que consideramos que se trata simplemente de formas aumentativas análogas a la que se da en librazo ('gran libro') o cuadernón ('gran cuaderno'):

(3) notición, estresazo, apurón, sustazo, miedazo, viajón, sorpresón, calorón, programón 
También dejamos de lado aquellos casos que implican un cambio de categoría respecto de la base, aun si son apreciativos. Así ocurre, por ejemplo, con la creación con -ón de nombres o adjetivos a partir de verbos:

(4) tragón, comilón, morfón, cagón, meón, reclamón, burlón, zumbón, jodón, mandón, mirón

Al tratarse de formas que implican el significado 'humano', las de (4) tienden a ser categorialmente ambiguas entre un adjetivo y un nombre. Se interpretan como '(el) que V mucho', por lo que solo pueden formarse a partir de verbos no delimitados que acepten la cuantificación y que, además, puedan constituir conductas prototípicas o caracterizadoras.

También son ambiguas desde el punto de vista categorial las formas de (5), parafraseables en su significado más literal (luego se producen lexicalizaciones) como 'que tiene un $\mathrm{N}$ grande' (que -según Lázaro Mora, 1999- constituía el valor original del sufijo en latín). Este valor está prácticamente circunscripto a la combinación de -ón con nombres que designen partes del cuerpo.

(5) culón, cabezón, narigón, panzón, tetona, culona, huevón, orejón, pintón ${ }^{3}$

\footnotetext{
${ }^{3}$ Alterna aquí -a veces- con - udo: cabezudo, tetudo, panzudo, orejudo, dientudo, narigudo (cfr. también, sin alternancia, boludo, buesudo, trompudo).
} 
En general, con el fin de evitar la ambigüedad, los hablantes parecen reservar -ón para esta interpretación peculiar con nombres de parte del cuerpo, y recurren a otro de los sufijos si se quiere expresar el significado más simple 'gran N' (que es el que focalizamos aquî): culote, cabezota, tetaza, bocaza, etc.

\section{I.1 Características morfológicas}

El sufijo -ón presenta una particularidad desde el punto de vista morfológico: al contrario de -ote y azo, que mantienen siempre el género de la base (cfr. 6.a), -ón es sistemáticamente masculino cuando se une a nombres de género arbitrario o invariable (i.e., sin flexión), por lo que puede modificar el género de la base (cfr. 6.b). Ese sufijo no presenta particularidades, sin embargo, al adjuntarse a palabras que flexionan en género, como adjetivos o nombres con género semántico, como muestran los casos de (6.c):

(6) a. puertota, orejota/ orejaza, tormentota, sillota, botellota, bocota/ bocaza

b. minón, patadón, mujerón, ofertón, remerón, alegrón, notición, peliculón

c. buenona, rubiona, compadrona, crudona, machona, muchachona 
Mientras que -ote, al igual que -ito, admite marginalmente la repetición del mismo sufijo (cfr. 7.a), la reaplicación de -a₹o u -ón en general no es posible en el español de Argentina. Una zona dialectal particular la constituye la provincia de Córdoba, en la que se encuentra un uso más libre de ambos sufijos: allí se produce la reaplicación productiva del mismo sufijo (-ón), al igual que en otras zonas americanas, como México o el Caribe (cfr. 7.b), además de una combinación muy productiva entre azo y -ón, que es inexistente o infrecuente en el resto de la Argentina (cfr. 7.c):

(7) a. grandotote, chiquitito

b. caloronón, buenonona

c. calorazononón, liazononón, negrazones

\section{I.2 Caracterización semántica}

Como ya se ha dicho, al igual que el resto de la morfología apreciativa, los sufijos aumentativos no dan lugar a nuevas entradas léxicas excepto que se produzca algún proceso inesperado de lexicalización. El significado que aportan parece más referencial en el caso de los nombres, ya que se liga con el tamaño físico o figurado de una entidad, persona o evento, $y$ mucho más evanescente con adjetivos, ya que pueden adoptar ahí desde valores efectivamente aumentativos hasta matices atenuativos, pasando por usos irónicos o despectivos. 


\section{i. Nombres}

En español rioplatense, los sufijos bajo análisis tienen sistemáticamente un significado aumentativo cuando modifican a nombres, independientemente de si estos designan objetos (8.a), personas (8.b) o eventos (8.c) ${ }^{4}$ :

(8) a. sillota, perrazo, gatote, autazo, ojazo, orejaza, librazo, librote, botellota

b. hombrote/ hombrón/ hombrazo, soldadote, minón, madraza, amigote, amigazo

c. viajón, calorón, miedazo, tormentón, batallota, fiestota, patadón, programón, partidazo ${ }^{5}$

En todos estos casos, la paráfrasis es 'gran' (i.e., 'gran auto', 'gran hombre', 'gran noticia'), pero la interpretación puntual será levemente distinta de acuerdo con el nombre involucrado:

${ }_{4}$ Si bien los sufijos aumentativos son muy productivos con nombres, parece haber algunas restricciones fonológicas adicionales para la formación, en paralelo a las bien estudiadas restricciones del diminutivo (cfr., por ejemplo, Ambadiang \& Camus (2012)). Así, parecen imposibles *trenazo o *trenón para tren, y aun la forma aceptada (trensote) es poco usada; en cambio, es posible el aumentativo con todos los demás nombres que designan vehículos: avionazo, autazo, colectivazo.

5 Cabe destacar que los nombres eventivos que se combinen con -ote/a son relativamente raros (se registran en Google tormentota o miedote, pero con pocas ocurrencias frente a las formas en -azo y -ón). Por su parte, -azo se combina preferentemente con nombres masculinos (así, se encuentran muy pocos eventivos femeninos en -aza: guerraza, tormentaza). 
así, el significado de 'gran' se interpreta como relativo al tamaño físico en algunos ejemplos de (8.a) y (8.b), pero es variable en el resto de los casos. En efecto, perrazo o librote indican tamaño físico, al igual que hombrote (también hombrón y hombrazo) o soldadote. En cambio, librazo se interpreta más bien como 'gran libro (para leer)' y minón como 'gran(=linda, atractiva) mina', mientras que otros casos son ambiguos (autazo puede hacer referencia al tamaño $o$ a la calidad indistintamente). Los nombres eventivos de (8.c), que son alternativamente simples o deverbales (aunque luego revisaremos algunas restricciones, cfr. los ejemplos de 18), requieren una interpretación más específica de 'gran' en función del nombre base involucrado: 'bueno, excelente' (programón, partidazo), 'importante' (notición, apurón), 'fuerte' (patadón), 'largo' (viajón), etc. ${ }^{6}$

Paradigmas paralelos podemos establecer para los prefijos de significado aumentativo, como re-, super-, hiper-, mega- y, en ocasiones, maxi- y macro- (ultra-, en cambio, parece limitado a los adjetivos):

(9) a. supersilla, superperro, reauto, megalibro, reárbol, superbotella, maxikiosco, macroestructura

\footnotetext{
${ }^{6}$ En términos de Pustejovsky (1995), puede decirse que el aumentativo modifica alternativamente distintos elementos de la entrada léxica (los qualia formal o télico, el aspecto, etc.).
} 
b. remadre, remédico, supermina, megaamigo, superviajero ${ }^{7}$

c. megaviaje, recalor, supermiedo, supertormenta, megafiesta, reprograma, megapartido, hiperespeculación

Los nombres creados con -ote, -azo y -ón suelen ser semánticamente opuestos a los mismos nombres con el afijo diminutivo -ito, sobre todo cuando se refieren a tamaño físico, sean objetos, como en casota vs. casita, perrazo vs. perrito, o nombres que designan seres humanos: hombrón/ hombrazo vs. bombrecito (cfr. 8.a-b con los ejemplos relevantes de 10.a-b). Lo mismo ocurre cuando los afijos modifican a nombres eventivos, con los que el diminutivo presenta distintas interpretaciones: 'pequeño', 'suave', 'corto', 'leve', en su mayoría opuestas a los diversos valores que puede adoptar 'gran': viajón vs. viajecito o miedazo vs. miedito (cfr. 8.c-10.c):

(10) a. sillita, perrito, gatito, autito, ojito, orejita, librito, botellita, casita, heladito

b. hombrecito, soldadito, mujercita, amiguito, madrecita, minita

c. viajecito, calorcito, miedito, tormentita, fiestita, patadita, programita, partidito

\footnotetext{
${ }^{7}$ En los casos de nombres que designan seres humanos, sin embargo, no es posible interpretar los prefijos con valor de tamaño físico: rehombre o supersoldado, que son posibles, no reciben la misma lectura de hombrote/ hombrazo o soldadote.
} 
Nótese, sin embargo, que no siempre significan lo contrario de las formas diminutivas, cuando el significado afecta la valoración del nombre: autazo o librazo no se oponen a autito o librito (cfr. 8.a-10.a), ni amigote y amigazo a amiguito, ni minón a minita, ni madraza a madrecita (cfr. 8.b-10.b), ni partidazo a partidito o programón a programita (cfr. 8.c-10.c)

Las similitudes de significado entre los sufijos aumentativos hacen que presenten frecuentes alternancias con las mismas bases nominales (cfr. 11.a). Sin embargo, en ocasiones hay un cambio de significado referencial o connotativo: es sistemático el que opone las formas en -ón de (5) a las formadas sobre las mismas bases con -ąo y -ote, que señalan tamaño físico (cfr. 11.b), mientras que en (11.c) se presenta un caso donde hay una marcada diferencia de significado entre ambas formas, con amigazo como la forma "auténticamente" aumentativa ('gran amigo') y amigote con un significado que puede adoptar valores despectivos o atenuativos ('medio amigo', 'no tan amigo'):

(11) a. heladote/ heladón, autazo/ autote, hombrón/ hombrote/ hombrazo, tormentota/ tormentón, quilombón/ quilombazo

b. culón vs.culazo, orejón vs. orejota/ orejazas, panzón vs. panzota

c. amigazo/ amigote 


\section{ii. Adjetivos}

En las descripciones morfológicas suele resaltarse el valor peyorativo original de los sufijos aumentativos en combinación con adjetivos, aunque también se registra el hecho de que en todo el español -y particularmente en las variedades americanas- se advierte un uso creciente con un valor predominantemente aumentativo (cfr. Lázaro Mora 1999), que sería paralelo al significado de tamaño que -ón, -azo y -ote aportan a los nombres con los que se combinan, según hemos visto anteriormente. Así, en formas como las de (12), el significado aportado por -azo y -ote se aproxima al de -isimo o los prefijos re-, super-, biper-, mega-, ultra-, al funcionar como intensificativos de la cualidad que modifican:

(12) a. negrazo, piolazo, buenazo, grandote, cobardote, tranquilazo, flojazo, lindazo ${ }^{8}$

${ }^{8}$ Las mismas formas tienen usos que no son aumentativos, sino que toman otros valores, a veces lexicalizados (como en buenazo por 'persona buena y débil de carácter'). Los siguientes ejemplos (consultados en las páginas web que aparecen como referencia el 6/3/2016) ilustran los usos más claramente aumentativos de esos sufijos:

(i) a. piolazo el tema para las chicas para que valoren a su pareja (https://plus.google.com/116061541753016314017/posts/JZzSThVryfq)

b. hoy estaba hablando con un amigo y le pasé una canción, le dije que la escuchara porque buenaza (https://ar.answers.yahoo.com/question/index?qid=20140826231523AAbgIYf)

c. Todos concordamos en que el vestido es negrazo negrazo (https://twitter.com/yullietmf/status/571259676551217152) 
b. supernegro, hiperpiola, rebueno, ultragrande, megatranquilo, superflojo, relindo

A pesar de que comparten el valor intensificativo, los sufijos aumentativos siempre tienen más connotaciones que los prefijos o que el superlativo: no se parafrasean simplemente como 'muy'. De hecho, en muchos casos, -ón, en especial, y a veces también -ote no aportan un significado aumentativo (ni tampoco peyorativo), sino predominantemente atenuativo:

(13) tristón, dulzón, simplón/simplote, payasón, seriote, secote, rubión

En estos casos, el significado que aporta el sufijo no es intensificativo de la cualidad; por el contrario, se interpreta como 'medio, un poco, más bien A'. En ese sentido, en los ejemplos de (13) los sufijos aumentativos funcionan como un recurso de atenuación, que supone una moderación cortés de una afirmación que podría tomarse como agresiva o lesiva por parte del hablante, en lo cual funcionan de modo semejante al afijo diminutivo -ito (según la descripción de Kornfeld (2015)). Hay, de hecho, algunas alternancias entre el afijo diminutivo y

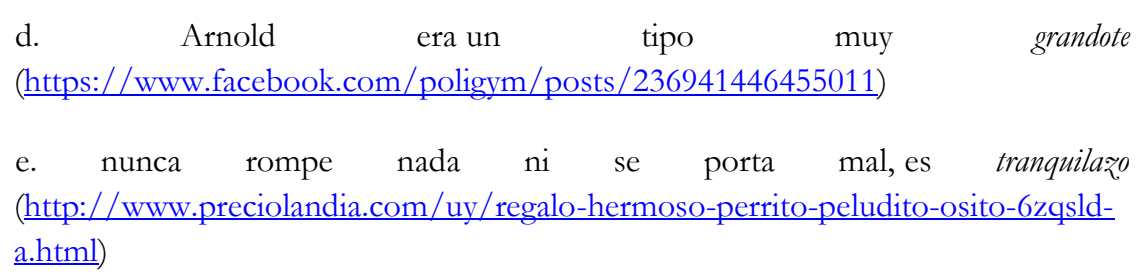


los sufijos aumentativos -ote y, sobre todo, -ón en combinación con los mismos adjetivos:

(14) amargón /amarguito, crudón / crudito, arruinadón/ arruinadito, apuradón / apuradito, seriote/ seriecito, cortón / cortito, cuadradote/ cuadradito, facilote/ facilito

Es decir que todos los ejemplos de (14), con sufijos aumentativos o diminutivos, agregan una atenuación afectiva a la base y no se advierten diferencias importantes de significado, sino valores sutiles (a veces, -ito es más despectivo o más afectivo que el correspondiente sufijo aumentativo, o a la inversa). Esta coincidencia diverge radicalmente del funcionamiento de los mismos afijos con los nombres, donde encontramos una sistemática oposición semántica entre -ito y los sufijos aumentativos en los nombres que designan objetos y eventos, mientras que con nombres que designan personas hay diferencias semánticas considerables, sin llegar a la franca oposición (ver ejemplos de 8 en contraste con 10).

Por otra parte, también en el caso de su combinación con adjetivos los sufijos aumentativos pueden alternar sin cambio de significado evidente en pares, como muestran los casos de (15):

(15) rubiote/ rubión, coloradote/ coloradón, negrón/ negrazo, morochote/ morochazo/ morochón, boludón/ boludazo/ boludote 
En suma, los sufijos aumentativos combinados con adjetivos pueden oscilar entre una interpretación intensificativa de la cualidad a la que se adjuntan (como es el caso con -azo y -ote, en 12.a) y una atenuativa (-ón y otra vez -ote en 13-14). De este modo, -ote tiene el curioso estatuto de un elemento que, de acuerdo con los estudios pertinentes (cfr., por ejemplo, Landone (2009)), puede representar dos polos opuestos en el terreno de la cortesía verbal.

\section{I.3 Distribución gramatical}

Una de las principales propiedades sintácticas, que además refiere a un elemento que es común a diversas clases de palabras, es el aspecto léxico. Al proponer que el rasgo [+/delimitado] es transcategorial, Morimoto (1998) subsume dos tradicionales oposiciones: la de nombres contables y de masa y la de adjetivos relacionales y calificativos. Ambas oposiciones resultan pertinentes para evaluar el comportamiento de los sufijos aumentativos con nombres y adjetivos, como repasaremos a continuación.

\section{i. Nombres}

En líneas generales, los nombres de masa no pueden recibir sufijos aumentativos (cfr. 16.a), excepto en el caso de algunos 
eventivos que también admitirían ser modificados por 'gran', como en (16.b): es posible un gran miedo, pero no *gran harina o * gran arena:

(16) a. *arenoza, *orazo, *harinota, *aguota, *petroléon, *azucarón 9

b. miedazo, estresazo, calorazo/ calorón, quilombón, alegrón

De todos modos, los casos de (16.b) no tienen un estatuto muy claro en relación con la delimitación: no siempre aceptan la modificación por los cuantificadores propios de los nombres contables (cfr. 17.a), pero, como se muestra en (17.b-c) tampoco pueden aparecer desnudos o con cuantificadores en singular, como se espera para los nombres de masa (cfr. Bosque (1999)). En ese sentido, se contraponen también al comportamiento de sus propias bases, como se especifica en cada caso:

(17) a. varios alegrones / quilombones; *varios calorazos / estresazos / miedazos

b. Se armó *(un) quilombón (cfr. Se armó quilombo), Hace *(un) calorazo (cfr. Hace calor); Me agarró *(un) estrezaso (cfr. Me agarró estrés); Había *(un) alegrón (cfr. Había alegría); Me dio *(un) miedazo (cfr. Me dio miedo)

9 Si hay ambigüedad entre la interpretación de masa y contable, el aumentativo desambigua en favor de la segunda lectura (cfr. naranjaza, pescadote). Una excepción es platota, intrínsecamente de masa, que muestra varias apariciones en Google. 
c. ?? un poco de quilombón (cfr. un poco de quilombo); ?? mucho calorazo (cfr. mucho calor); ?? tanto estresazo (cfr. tanto estrés); ?? bastante alegrón (cfr. bastante alegría); ?? demasiado miedazo (cfr. demasiado miedo)

Cabe señalar que, a su imposibilidad de combinarse con nombres de masa, se suman diversas restricciones de los sufijos aumentativos en su relación con ciertos sufijos deverbales, como -ción, -miento, -aje, -dor (cfr. los ejemplos de 18.a), deadjetivales, como -(i)dad, -ia, -eza, -ura (cfr. 18.b) y denominales, como $-i s t a^{10}$, típico de las profesiones o los partidarios de alguna doctrina, o con los casos de composición culta o nativa (cfr. 18.c-d):

a. *contaminacionota, *abatimientón, *aterrizajazo, *batidorota

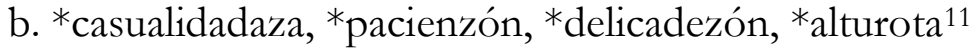

c. *taxistazo, *lingüistón, ${ }^{*}$ pacifistote, ${ }^{*}$ peronistazo

d. *filosofón, *antropologazo, *cuidacochote, *alcanzapelototas ${ }^{12}$

10 En forma paralela a -ito, sí pueden aparecer casos de nombres de profesiones formadas con -ote o -azo a partir de sufijos denominales como -ero/a o -al (e.g., arquerazo. costurerota, obrerazo, colectiverote, intelectualazo) o de sufijos deverbales como -dor (trabajadorazo, cazadorazo) o las formas participiales (empleadote).

11 En cambio, los prefijos aumentativos pueden combinarse sin problemas con cualquiera de esos sufijos:

(i) a. recontaminación, superabatimiento, megaaterrizaje, maxibatidora

b. recasualidad, hiperpaciencia, megadelicadeza, superaltura 


\section{ii. Adjetivos}

Como se puede ver en los ejemplos relevantes anteriores (por ejemplo, 12-15), los sufijos -ón, -azo y -ote se combinan básicamente con adjetivos calificativos. En otros términos, esos sufijos resultan ser absolutamente incompatibles con adjetivos relacionales y adverbiales, es decir con adjetivos que tengan el rasgo [+delimitado]:

(19) a. *sintagma nominalote, *producción pesqueraza, *postura filosoficota, *gusto culinarión, *lesión cutaneaza

b. *futurote presidente, *aparentaza dificultad, *continuón ruido, *antiguazas murallas

Pero además, y en forma paralela a lo que acabamos de resaltar en relación con los nombres, tampoco se aplican a una serie de adjetivos calificativos derivados de los sufijos deverbales $-d o r,-n t e,-b l e,-i v o,-i z o$ :

(20) -dor: *seductorsote, *ansadorazo, *ensordecedorón, *arrulladorazo

${ }^{12}$ Hay otros nombres de profesión con los que tampoco parece posible la aplicación de sufijos aumentativos: $\mathrm{cfr}$. ??/*arquitectazo, cientificazo. 
-nte: *constantote, *atrayentazo, *entusiasmantón, *perseverantón

-ble: *amabilazo, *agradabilón, *pasabilote, *envidiabilote

-ivo: *destructivazo, *constructivote, *llamativón, *atractivazo

-izo: *movedizón, *resbalidizote, *quebradizazo, *olvidadizón

Cabe resaltar que, al ser calificativos, los adjetivos de (20) son perfectamente compatibles con cuantificadores sintácticos de grado: muy constante, bastante seductor, tan amable, demasiado destructivo, sumamente movedizo, etc.

\section{Análisis y discusión}

A continuación pondremos en relación las observaciones descriptivas anteriores sobre -ote, -azo y -ón con las propiedades de otros afijos apreciativos.

i. En forma paralela a los ejemplos de (20), el afijo -ito no puede combinarse con adjetivos calificativos derivados de los sufijos deverbales $-d o r,-n t e,-b l e,-i v o,-i \not 0^{13}$. En Kornfeld

${ }^{13}$ En este punto se asemejan al diminutivo:

(i) a. *seductorcito, ${ }^{*}$ cansadorcito, ${ }^{*}$ ensordecedorcito, *arrulladorcito

b. ${ }^{*}$ constantito, *atrayentito, *entusiasmantito, * perseverantito

c. *amabilito, *agradabilito, *pasabilito, *envidiabilito

d. $*$ destructivito, ${ }^{*}$ constructivito, *llamativito, *atractivito

e. ${ }^{*}$ movedicito, ${ }^{*}$ resbalidicito, ${ }^{*}$ quebradicito, ${ }^{*}$ olvidadicito, ${ }^{*}$ enamoradicito 
(2015) se observa que hay restricciones ulteriores en la distribución del diminutivo, como muestra el siguiente contraste:

(21) a. inutilito, rapidito, cobardito, tristecito, vivito, piolita, feíto

b.??utilito,??velocito,??valentito,??alegrito,??lealito,??inteligentito,? ?astutito,??bellito

Nótese que los adjetivos de (21.a) y (21.b) son próximos desde el punto de vista del significado: se trata de (cuasi) sinónimos, antónimos o se ubican en el mismo campo semántico. Cabe destacar que los adjetivos calificativos que pueden combinarse con -ito (no derivados de -dor, -nte, -ble, ivo o -izo) son los mismos que pueden recibir modificadores como medio, un tanto, algo o un poco, como se puede comprobar en el perfecto paralelismo entre (21) y (22):

(22) a. Es medio inútil / rápido / triste / cobarde / vivo / piola $/$ feo

b. *Es medio útil / veloz / alegre / valiente / leal / inteligente / astuto / bello

En cambio, los sufijos aumentativos son generalmente malos en combinación con adjetivos derivados en -oso (con alguna excepción, cfr. asquerosón/ asquerosote), que sí dan lugar productivamente a formas diminutivas:

(ii) a. tramposito, vanidosito, graciosito, esponjosito, perezosito, celosito

b. *tramposazo, *vanidosote, *graciosón, *esponjosazo, *perezosote, *celosazo 
Esta distribución puede interpretarse como resultado del carácter atenuador del diminutivo, en lo que coincide con medio, un tanto, algo o un poco. Los adjetivos que designan cualidades puramente positivas en general no pueden ser modificados por esos recursos porque resultaría incongruente que el hablante los seleccione para luego morigerarlos; cuando son posibles, remiten a un significado irónico o puramente afectivo $^{14}$.

En ese sentido, los sufijos aumentativos se combinan con los mismos adjetivos que -ito y medio:

(23) a. inutilón, rapidón, cobardón, tristón, vivazo, piolazo, feote

${ }_{14}$ En los siguientes ejemplos (consultados en las páginas web que aparecen como referencia el 6/3/2016) se advierten usos irónicos (i) y puramente afectivos (ii), de las formas que hemos señalado como infrecuentes:

(i) a. crea esta casta abyecta de cientificos arrogantes, tan inteligentitos que son (http://evolucionarios.blogalia.com/historias/32463)

b. no le permito que me llame inteligentisimo, jovencito. Apenas inteligentito y hasta por ahi nomas (http://weblogs.clarin.com/eltoquemactas/2012/01/13/multitasking/)

c. Che, ese astutito sonó re agresivo (http://lapiedralibre.blogspot.com.ar/2008/02/mi-familia-es-un-dibujo$\underline{1123 . h t m l})$

d. La cuestión es que Clay estuvo muy astutito con todo esto (http://www.meh-charlas.com/2013/09/soa-602-one-one-six.html)

(ii) a. Eres mi precioso bellito te necesito y te amo (http://santiagoramundo.com/entrevistas/)

b. Holaa a todos yo soy lo mas bellito de mi familia, Anny (http://twicsy.com/i/JVGnXh)

c. patito hermosito (https://m.ask.fm/loslorosvieja)

d. sos hermosito, te amo

(https://twitter.com/micafrasson/status/364513346508169216) 
b. ??utilote, ??velozón, ??valentote, ??alegrazo, ??inteligentote, ??astutazo, ??bellote 15

Los datos de (21-23) sugieren que hay consistencia en la división señalada en Kornfeld (2015) entre los adjetivos calificativos que pueden combinarse con modificadores como medio, un tanto, algo o un poco y que serán también los que reciban la afijación con -ito, -ote, -azo, -ón.

En este punto, tanto el diminutivo como los aumentativos se diferencian de los prefijos equivalentes como re-, super-, mega-, hiper- (y también el "superlativo" -isimo), cuyas posibilidades combinatorias incluyen a todos los adjetivos calificativos que puedan combinarse también con modificadores sintácticos de grado como muy, bastante, poco, tan, etc.

Es interesante observar al respecto que los cuantificadores sintácticos como muy, bastante, demasiado, tienen una distribución complementaria con los prefijos y el superlativo:

(24) a. ??muy lindísimo, ??demasiado tontísimo

b. ??demasiado relindo, ??tan superbajo

${ }^{15} \mathrm{Si}$ existen formas análogas a (23.b) (inteligentazo, por ejemplo, se encuentra con varias ocurrencias en Google), sistemáticamente solo tienen un valor aumentativo "puro", equivalente a 'muy', o bien suponen una interpretación irónica (y, por lo tanto, negativa), como en valentón. 
En cambio, esos cuantificadores de grado sí pueden combinarse, en general, con los sufijos aumentativos (y también con el diminutivo):

(25) muy grandote, demasiado buenazo, bastante tristón

Del contraste entre estos ejemplos puede deducirse que solo el superlativo y los prefijos de (24) funcionan como núcleos del Sintagma de Grado, y por eso no aceptan coaparecer con cuantificadores sintácticos que ocupan la misma posición. En cambio, los sufijos apreciativos de (25) se ubicarían en una posición más cercana a la raíz léxica, en términos de la Morfología Distribuida (cfr. Marantz (2001) o Embick \& Halle, (2011)), lo cual explica, de paso, sus mayores irregularidades (cfr., más adelante, la estructura de 41).

ii. Ya hemos establecido el paralelismo entre los pares de sufijos aumentativos -ote, -azo, -ón y los prefijos "análogos" re-, super-, mega-, hiper- aplicados a nombres (cfr. 8-9) y adjetivos (cfr. 12). A continuación recuperamos ese paralelismo, en el que tanto sufijos como prefijos se interpretan como 'muy' combinados con adjetivos (dejando de lado usos atenuativos, despectivos o puramente afectivos) y, combinados con nombres, se interpretan como 'gran' (o 'buen'); de hecho, encontramos pares prácticamente sinónimos de adjetivos (26) y nombres (27): 
(26) tranquilazo / supertranquilo, grandote / megagrande, flojazo / reflojo

(27) a. un autazo / un reauto, un librazo / un relibro, un arbolote / un reárbol

b. un minón / una remina, un amigazo / un reamigo, una madraza/ una supermadre

c. un calorón / un recalor, un viajón / un reviaje, un quilombazo / un requilombo

Sin embargo, en Kornfeld \& Kuguel (2013) se observa que la distribución de $r e$ - en español rioplatense es mucho más amplia que la de -ote, -azo, -ón, ya que aparece (con un significado perfectamente predecible desde el punto de vista gramatical) también con verbos no delimitados, con una interpretación cuantificacional ('mucho', como en 28.a) o durativa ('mucho tiempo', como en 28.b), y, con verbos delimitados, con una paráfrasis iterativa ('muchas veces', como en 28.c) o enfatizadora de la delimitación o culminación del evento ('hace mucho' o 'completamente', como en 28.d).

(28) a. se reenoja, le reteme, lo requiere

b. retrabaja, rejuega, reesperó, redurmió

c. lo rebesa, reviajó a Europa, reva al cine

d. reterminó la reunión, rellegó, se redurmió. 
Nótese que, en estos contextos, también podría aparecer super- (e.g., se superenoja, lo superbesa, supertrabaja), que, en cambio, no puede alternar en el uso de re-como modificador modal de toda la oración, cuyos valores se ligan con la certeza absoluta (29.a) o, en las nuevas generaciones, con una negación irónica (29.b-c):

(29) a. re llega a tiempo, Re que lo hago para quedar bien.

b. A: ¿¿Qué opinás de Javier?

B: - iLo odio! Ah re que es mi mejor amigo.

c. A: $-¿$ Cómo te fue en el parcial?

B: -Genial. Ah re que me saqué un dos.

En Kornfeld \& Kuguel (2013) se distinguen el caso del recuando se une a adjetivos, verbos y oraciones sobre la base de que en esos casos el afijo puede modificar frases (cfr. 30) y constituir enunciados independientes (cfr. 31):

(30) a. re [lindo e inteligente]

b. Juan re [jugó y trabajó]

(31) a. -¿Es lindo?

- Re.

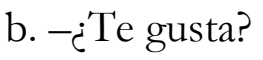

- Re.

c. - ¿Venís? 
- Re.

Los datos de (30-1) justifican el tratamiento de re- como un elemento que morfofonológicamente tiene un estatuto intermedio entre una palabra y un clítico y sintácticamente es núcleo de una proyección funcional, que tentativamente podemos ubicar como Sintagma de Grado, Sintagma de Aspecto o algún sintagma ligado con la modalidad entre las proyecciones superiores de la cláusula, como el Sintagma de Fuerza (Rizzi, 1997).

Ese comportamiento sintácticamente transparente no es posible en el caso de la combinación de re- con nombres (cfr. 32), lo cual lleva a considerar que en ese caso se trata de un "verdadero" afijo, más cercano a la raíz léxica, en el sentido de Marantz (2001) o Embick \& Halle (2011):

(32) a. *re [auto y compu]

b. - ¿ंTiene una casa?

-\#Re [solo con el significado de 'seguro', es decir modal]

Los sufijos aumentativos no permiten ningún fenómeno de transparencia sintáctica, ni con adjetivos (33) ni con nombres (34):

(33) a. *[buen y trist]ón

b. - ¿Está triste? 
*_Ón

(34) a. * auto y cas]ota

b. -¿'Tiene una casa?

*_Ota

Los ejemplos de (33) y (34) sugieren que también los aumentativos ocupan una posición cercana a la raíz léxica.

iii. Finalmente, y a modo de acotación marginal, un dato que diferencia a los sufijos aumentativos del diminutivo -ito es la posibilidad de este último de combinarse con nombres de masa, en contraste con lo que ya hemos visto que ocurre con la agramaticalidad de los mismos nombres con sufijos aumentativos en (16.a):

(35) arenita, agüita, harinita, orito, arrocito, azuquita (azuquítar), papita, cafecito

Hemos indicado también que la agramaticalidad de los ejemplos de (16.a) resulta esperable porque, para que una entidad tenga tamaño ('grande' o 'pequeño') debe contar con límites precisos y no ser meramente una sustancia. En ese sentido, debe entenderse que la mayor "flexibilidad" del diminutivo responde a que -ito puede modificar a todo un enunciado, y no únicamente a la palabra a la que se adjunta. Así, a menudo -ito actúa como un recurso atenuador o bien del 
acto de habla (pedido, orden, sugerencia, etc.), como en (36), o bien del contenido proposicional (37.a-b), lo que obviamente puede derivar luego en otra clase de interpretación (irónica, afectiva, peyorativa) (cfr. 37.c):

(36) a. ¿No tiene un cigarrito?

b. ¿'Tomamos (un) cafecito?

(37) a. Me dio miedito.

b. ¿Puedo salir un ratito?

c. Te va a costar mucha platita.

Como es evidente, los sufijos aumentativos no pueden dar lugar a oraciones como las de (36-7), al menos con la interpretación relevante.

iv. Para dar cuenta estructuralmente de las diferencias que acabamos de reseñar, partimos dentro de la Morfología Distribuida de la propuesta de Marantz (2001), retomada y sistematizada por Embick \& Halle (2011). El supuesto de Marantz es que las raíces léxicas no tienen especificada su categoría per se, sino que esta se adquiere en combinación con las llamadas "categorías chiquitas" ( $n, v, a)$, que nuclean proyecciones funcionales destinadas (precisamente) a categorizarlas. Se vinculan a esas proyecciones $\mathrm{S} n, \mathrm{~S} v$ y $\mathrm{S} a$ los morfemas derivativos; así, una forma compleja como 
nominalización constará de una raíz nomin acompañada por sucesivas proyecciones categoriales: yendo de la más incrustada a la más superficial, $\mathrm{S} a, \mathrm{~S} v, \mathrm{~S} n$ (en las que los morfemas derivativos serán los núcleos, dado que aportan las sucesivas categorías del conjunto):

(38) $\left[\mathrm{s}_{n}-\right.$ ción $\left[\mathrm{s} v-\right.$ iza $\left.\left[\mathrm{s}_{a}-\mathrm{al}[\mathrm{VNOMIN}]\right]\right]$

Cuanto más alejada esté una proyección funcional de la raíz, más transparente será su funcionamiento sintáctico, predice Marantz; cuanto más próxima, más "irregular" o idiosincrásico será. Esto explica las particularidades de los morfemas descriptos tradicionalmente como derivativos, cuyo carácter más "léxico", "irregular" o "idiosincrásico" que los morfemas flexivos es una simple consecuencia de su posición sintáctica, más próxima a la raíz léxica.

A partir de ese marco teórico, podemos proponer una serie de configuraciones que permitirían reflejar el comportamiento "transparente" de re- en el ámbito adjetivo y verbal: en ambos casos, suponemos que encabezaría una proyección funcional alejada de la raíz léxica, es decir, independiente del correspondiente sintagma categorial, $\mathrm{S} a$ o $\mathrm{S} v$. En (38.a) se ilustra cómo ese prefijo ocupa la posición de núcleo de un Sintagma de Grado en una secuencia como re débil, dando cuenta de la distribución complementaria con cuantificadores sintácticos y de la posibilidad de combinarse con cualquier adjetivo que lleve el rasgo [-delimitado] en $a^{\circ}$ (es decir, que sea calificativo), mientras que (38.b) exhibe un comportamiento 
perfectamente paralelo en relación con la cuantificación verbal, como en (lo) re ama:

(39) a. [sGrado [Grado re- [sa [a -delimitado [NDÉBIL]]]]]

b. [sCuant $\left[\right.$ Cuant' $r e-\left[\mathrm{s}^{2}\left[v^{v}\right.\right.$-delimitado [VAMAR $\left.\left.\left.\left.]\right]\right]\right]\right]$

El hecho de que re- encabece sendas proyecciones funcionales explica, adicionalmente, su enorme transparencia sintáctica, que hemos vista reflejada en los datos de (30-31).

Por su parte, de los datos de (32-33), así como de las restricciones combinatorias de los sufijos con nombres (véase ejemplos 18), se deduce que, al igual que los sufijos -ote, -azo y -ón, re- ocupa una posición mucho más cercana a la raíz léxica en el ámbito nominal. La configuración de (39) permite derivar indistintamente re auto o autaro, que tendrían la misma estructura; a diferencia del ejemplo de (38), los afijos en este caso ocupan un lugar de especificadores de la proyección categorial, en la medida en que no son responsables de la categoría de la palabra:

(40) $\left[\mathrm{s}_{n} r e-/-a q_{0}\left[n^{n}+\right.\right.$ delimitado [vAUTO $\left.]\right]$

Un esquema muy semejante al de (40) debemos proponer para dar cuenta de la combinación de sufijos aumentativos con adjetivos, como en grandote. La configuración de (40), con el sufijo en la posición de especificador de la proyección 
categorial $\mathrm{S} a$, se diferencia radicalmente de la propuesta en (39.a) para re-, dando cuenta de las mayores restricciones combinatorias de los sufijos aumentativos y de las idiosincrasias en el significado de la palabra resultante:

(41) $\left[\mathrm{s}_{s}-\right.$ ote $\left[{ }^{a}\right.$-delimitado [ $\left.\left.\left.\mathrm{VGRANDE}\right]\right]\right]$

En suma, en un esquema como el de Marantz, la diferencia de comportamiento entre afijos apreciativos que hemos ilustrado aquí, en particular en los ejemplos de (30-34), puede derivarse del hecho de que la posición de especificador de una proyección categorial $\mathrm{S} x$ difiere radicalmente de la posición de núcleo de una proyección funcional superior, como Sintagma de Determinante o Sintagma de Cuantificación. En el primer caso, en la combinación entre elementos funcionales (en este caso los afijos) y léxicos sobrevendrán significados "negociados", enciclopédicos y arbitrarios, acompañados de comportamientos sintácticos igualmente opacos; en el segundo, en cambio, obtendremos significados regulares y composicionales, así como una transparencia absoluta en términos sintácticos.

\section{Conclusiones}

Desde el punto de vista sintáctico-formal, a partir de lo discutido en este trabajo puede afirmarse que los sufijos aumentativos -ote, -ąo y -ón serían siempre "verdaderos 
afijos", sin propiedades que se proyecten en la frase o la oración (como sí ocurre con los prefijos re- o super-). Ello explica sus fuertes restricciones en la combinación con nombres (cfr. ejemplos de 18) y adjetivos (cfr. ejemplos de 20 y 23), así como su distribución no complementaria con modificadores de grado (cfr. 25). Hacia el final del artículo hemos propuesto un análisis en términos de la Morfología Distribuida (cfr. Marantz (2001) o Embick \& Halle (2011)). Podemos concluir que los sufijos apreciativos ocupan siempre una posición cercana a la raíz léxica. Se comportan, así, en forma contraria a lo que ocurre con el re- que se combina con adjetivos o verbos, cuyo comportamiento regular y previsible justifica su tratamiento como un elemento que puede encabezar en la sintaxis un Sintagma de Grado o de Cuantificación.

En cuanto a los factores semántico-pragmáticos que intervienen en su distribución, a lo largo del trabajo hemos podido comprobar que el empleo de los sufijos aumentativos no parece motivado en general por la función referencial (excepto en casos muy lexicalizados como palabrota o corralón, o cuando el sufijo transmite un significado "puro" de tamaño, como en camperón), sino por otras funciones "menores": la poética, la conativa y la emotiva (Jakobson, 1960). Como hemos dicho ya en el inicio del trabajo, en la propia idea de morfología apreciativa está implícita la función emotiva, que hace hincapié en el hablante, ya que supone siempre una marca de subjetividad. Se entrevera la función conativa, centrada en el oyente, que se observa en las múltiples y divergentes relaciones interpersonales: la atenuación cortés (Es medio secote), la 
afectividad (Es una madrazal un minón) o la ironía (Mejor cerrá tu bocota, Es un ladronazo). Por último, prima la función poética cuando el juego sobre el significante (y por lo tanto el peso de factores estéticos o estilísticos) se vuelve fundamental, como claramente ocurre en los fenómenos de repetición de los sufijos o de selección de uno sobre otro por motivos prosódicos o rítmicos ${ }^{16}$.

${ }^{16}$ En esa distribución, los sufijos coinciden, desde ya, con el resto de la morfología apreciativa, pero también con otros fenómenos denominados misceláneos (cfr. Lang, 1990) por quedar fuera de la morfología propiamente dicha: acortamiento (cole, profe, milico, monto, chori), "paranomasia" (lenteja (>lento), cortina (>corto), durazno (>duro)) y "vesre" (yeca, grone, feca, garcar, tordo). 


\section{Referencias bibliográficas}

Ambadiang, T. \& Camus, B. (2012). "Morfofonología de la formación de diminutivos en español: ¿reglas morfológicas o restricciones fonológicas?". Fábregas, A. et alii (Eds.). Los límites de la Morfología. Estudios ofrecidos a Soledad Varela Ortega. Madrid: UAM, pp. 55-78.

Benveniste, É. (1974). “El aparato formal de la enunciación”. Problemas de lingüística general II. Buenos Aires: Siglo Veintiuno, pp. 82-91.

Bosque, I. (1999). “El nombre común”. Bosque, I. \& Demonte, V. (Eds.). Gramática Descriptiva de la Lengua Española. Madrid: Espasa Calpe, pp. 3-77.

Bosque, I. \& Masullo, P. (1996). "On verbal quantification in Spanish". Proceedings of Third Workshop on the Syntax of Central Romance Languages. Girona: Universitat de Girona, pp. 9-63.

Embick, D. \& Halle, M. (2011). Word Formation: Aspects of the Latin Conjugation in Distributed Morphology. Berlin: Mouton de Gruyter.

Jakobson, R. (1988) [1960]. "Lingüística y poética”. Ensayos de lingüística general. Madrid: Cátedra, pp. 27-75.

Kornfeld, L. (2015). "Una propuestita astutita: el diminutivo como recurso atenuador". Artículo presentado para su evaluación en la Revista Iberoamericana de Lingüística (RILI) en octubre de 2015.

Kornfeld, L. \& Kuguel, I. (2013). "Un afijo re loco (Notas sobre re)”. Di Tullio, A. (Ed.). El español de Argentina: estudios gramaticales. Buenos Aires: EUDEBA, pp. 13-33.

Landone, E. (2009). Los Marcadores del Discurso y la Cortesía Verbal en Español. Berna: Peter Lang.

Lang, M. (1990). Formación de palabras en español. Madrid: Cátedra. 
Lázaro Mora, F. (1999). "La derivación apreciativa”. Bosque, I. \& Demonte, V. (Eds.). Gramática Descriptiva de la Lengua Española. Madrid: Espasa, pp. 4647-4682.

Marantz, A. (2001). "Words and things". Ms., MIT.

Morimoto, Y. (1998). El aspecto léxico. Madrid: Arco/Libros.

Pustejovsky, J. (1995). The generative lexicon. Massachussets: MIT Press.

Rizzi, L. (1997). "The fine structure of the left periphery". Haegeman, L. (Ed.). Elements of Grammar. Dordrecht: Kluwer.

Zacarías Ponce de León, R. (2008). "Morfemas apreciativos del español: entre la flexión y la derivación”. Núcleo, 25, pp. 221 $-237$.

Fecha de recepción: 3/11/2015

Fecha de aprobación: 9/3/2016 